\title{
Coupling of morphology to surface transport in ion-beam-irradiated surfaces: normal incidence and rotating targets
}

\author{
Javier Muñoz-García ${ }^{1}$, Rodolfo Cuerno ${ }^{2}$ and Mario Castro ${ }^{3}$ \\ ${ }^{1}$ Grupo Interdisciplinar de Sistemas Complejos (GISC) and School of Mathematical Sciences \\ and Complex and Adaptive Systems Laboratory, University College Dublin, Belfield, \\ Dublin 4, Republic of Ireland \\ ${ }^{2}$ Departamento de Matemáticas and GISC, Universidad Carlos III de Madrid, Avenida de la \\ Universidad 30, E-28911 Leganés, Spain \\ ${ }^{3}$ GISC and Grupo de Dinámica No Lineal (DNL), Escuela Técnica Superior de Ingeniería \\ (ICAI), Universidad Pontificia Comillas de Madrid, E-28015 Madrid, Spain \\ 口 \\ प
}

\begin{abstract}
Continuum models have proved their applicability to describe nanopatterns produced by ion-beam sputtering of amorphous or amorphizable targets at low and medium energies. Here we pursue the recently introduced 'hydrodynamic approach' in the cases of bombardment at normal incidence, or of oblique incidence onto rotating targets, known to lead to self-organized arrangements of nanodots. Our approach stresses the dynamical roles of material (defect) transport at the target surface and of local redeposition. By applying results previously derived for arbitrary angles of incidence, we derive effective evolution equations for these geometries of incidence, which are then numerically studied. Moreover, we show that within our model these equations are identical (albeit with different coefficients) in both cases, provided surface tension is isotropic in the target. We thus account for the common dynamics for both types of incidence conditions, namely formation of dots with short-range order and long-wavelength disorder, and an intermediate coarsening of dot features that improves the local order of the patterns. We provide for the first time approximate analytical predictions for the dependence of stationary dot features (amplitude and wavelength) on phenomenological parameters, that improve upon previous linear estimates. Finally, our theoretical results are discussed in terms of experimental data.
\end{abstract}

\section{Introduction}

There is a long tradition for the continuum description of the morphological dynamics of a solid surface undergoing ion-beam sputtering (IBS) (see [1] and references therein). While more traditional approaches employed a macroscopic viewpoint, the current availability of surface measurement techniques of ever-increasing spatial and temporal resolutions has driven the formulation of continuum descriptions that incorporate micro- or mesoscopic information. The pioneering work in this respect is due to Bradley and Harper (BH) [2] who, building upon Sigmund's description [3] of the statistics of collision cascades in amorphous targets at low to medium energies, derived an evolution equation for the surface height. Such a formulation allowed them to predict the orientation for ripple formation as a function of the angle of incidence, and other experimental features of pattern formation by IBS. The work of BH, while remarkably successful, has a number of limitations (see a review in [4]) that fostered further theoretical work in order to improve upon it (for a review, see [5]). We 
will collectively refer to these as one-field models, in which a single function describes the physical state of the system, namely the field $h(\mathbf{r}, t)$ providing the target height value at every point $\mathbf{r}$ above a reference plane, at time (fluence) $t$. These models have found an increased interest after the experimental verification [6-8] of the capabilities of IBS as a surface nanostructuring technique, in particular for the production of quantum dots. This type of pattern formation requires either normal incidence, or else oblique incidence onto rotating targets [5].

When applied to describing quantum dot production by IBS, one-field models have met a number of limitations. Leaving aside the case of targets that remain crystalline under irradiation (mostly metals, see [9]) and focusing on amorphous or amorphizable targets (semiconductors and insulators [5]), one-field models predict a surface morphology that is disordered and rough at long fluences, contradicting the experimental observation of large domains of ordered nanostructures (dots). Moreover, such models, being based on the Kuramoto-Sivashinsky (KS) equation [10] and its anisotropic and noisy generalization $[11,12]$, they do not seem able to account for the strong intermediate coarsening of dot characteristics (amplitude $A$ and wavelength $l$ ) that is frequently observed in these experiments. Moreover, wavelength coarsening is a fully nonlinear effect: given the analytical complexity of nonlinear systems, one-field predictions for the dependence of $A$ and $l$ on phenomenological parameters have remained to date restricted to linear estimates, which naturally may lack even qualitative reliability for experiments in which coarsening is actually observed. Another challenge for theoretical models is the dependence of some of the mentioned observables (like $A$ and $l$ ) on physical variables such as energy, fluence or temperature. Many of the former theories are unable to describe the mentioned dependence.

Physically, one-field descriptions correspond to a simplification by which the complex microscopic dynamics is encoded in that of the single height field. In particular, this requires relaxation effects other than sputtering, such as surface diffusion, to be incorporated in an ad hoc fashion. Moreover, they usually neglect redeposition effects, known to play a role in, for example, the correct prediction of sputtering yields [13]. Recently, a different class of models has been introduced [5, 14-18] in which the evolution of the height is coupled to that of a second field $R(\mathbf{r}, t)$, which describes the density of mobile species that implement actual material transport at the surface ${ }^{4}$. For targets on which defects pileup with continuing irradiation, a thin surface amorphous layer develops, across which viscous flow can eventually occur, as, for example, in the case of silica [19]. In such cases, $R$ can be approximately taken as the actual thickness of that layer. Although originally inspired in continuum descriptions of macroscopic pattern formation on granular systems (aeolian and underwater sand dunes), analogous two-field descriptions have been also employed for nanoscopic systems, such as, for example, growth by MBE (see [20] for a review).

\footnotetext{
${ }^{4}$ Below, $R$ will be taken with physical dimensions of height. Transforming a surface density field to a height field is achieved simply through multiplication by the atomic volume.
}

Recent work [5, 16-18] on two-field models of IBS has highlighted their capabilities to describe a large number of qualitative features of the experiments-such as in-plane ordering, wavelength coarsening, non-uniform ripple motion, etc-linking them to the main phenomenological parameters describing the experimental conditions. To date, the most detailed studies [5, 18] have focused on the more general case of oblique incidence and the ensuing ripple formation. In this paper, we focus on dot formation in which in-plane symmetry is restored, namely for normal incidence or for oblique incidence onto rotating targets. We will provide details on the derivation of the effective height equations describing the pattern formation near instability threshold and will study them numerically and analytically. Thus, we are not merely deriving a mathematically equivalent model for normal or oblique incidence but we are also focusing on the link between theory and experiment. More specifically, we provide for the first time approximate analytical predictions for the dependence of stationary dot features (amplitude and wavelength) on phenomenological parameters, which improve upon previous linear estimates. Finally, our theoretical results are compared with experimental data.

\section{2. 'Hydrodynamic' model}

Given the above definition of height $h$ and mobile layer thickness $R$, their dynamics are coupled as

$$
\begin{gathered}
\partial_{t} R=(1-\phi) \Gamma_{\mathrm{ex}}-\Gamma_{\mathrm{ad}}+D \nabla^{2} R, \\
\partial_{t} h=-\Gamma_{\mathrm{ex}}+\Gamma_{\mathrm{ad}},
\end{gathered}
$$

where $\Gamma_{\mathrm{ex}}$ and $\Gamma_{\mathrm{ad}}$ are, respectively, the rates at which material is dislodged from the immobile target due to ion bombardment (locally decreasing the value of $h$ ) and the rate at which mobile material incorporates back into the immobile bulk (locally increasing the value of $h$ ). In the equation for $R$, we have assumed that mobile material diffuses at the surface with surface diffusion constant $D$. Note, we assume no spontaneous (beam-independent) 'evaporation', so that we neglect evaporation/condensation effects [21, 22] (equivalently, we are assuming that the pressure in the vapor phase is negligible). The dislodged material may be either sputtered away or added back to the mobile thickness $R$ with an efficiency $(1-\phi) \equiv \bar{\phi}$. Thus, the fraction of atoms actually sputtered is represented by $\phi$ so that, for $\phi \neq 1$, local redeposition is partially allowed. Physically, such an addition back to the mobile layer corresponds mostly to material which has not received the right energy and/or momentum to be sputtered away, but which is actually dislodged from the immobile substrate, so that it becomes available to transport. For $\phi=1$ all eroded atoms are sputtered away, while in the $\phi=0$ case the sputtering yield is zero. In the last case the effect of the ion beam is simply to provide material for surface transport while conserving the total mass. We will refer to the latter two cases as zero-redeposition and completeredeposition limits, respectively. They will constitute useful limiting cases below. 
In general, the rates of excavation and addition are necessarily functions of the surface morphology and the thickness of material transport (namely, of the space derivatives of $h$ and $R$, respectively). For arbitrary angles of incidence ${ }^{5}$, the excavation rate $\Gamma_{\text {ex }}$ reflects the lack of in-plane symmetry and, to lowest orders in height derivatives, has the shape

$$
\begin{aligned}
\Gamma_{\mathrm{ex}} & =\alpha_{0}\left[1+\alpha_{1 x} \partial_{x} h+\nabla \cdot\left(\underline{\alpha_{2}} \nabla h\right)+\partial_{x} \nabla \cdot\left(\underline{\alpha_{3}} \nabla h\right)\right. \\
& \left.\left.\left.+\sum_{i, j=x, y} \alpha_{4 i j} \partial_{i}^{2} \partial_{j}^{2} h+\partial_{x} h \nabla \cdot \underline{\left(\alpha_{5}\right.} \nabla h\right)+\nabla h \cdot \underline{\left(\alpha_{6}\right.} \nabla h\right)\right],
\end{aligned}
$$

where $\alpha_{i}=\operatorname{diag}\left(\alpha_{i x}, \alpha_{i y}\right)$ are $2 \times 2$ diagonal matrices for $i=$ $2,3,5, \overline{6}$. While the parameter $\alpha_{0}$ provides the rate of erosion for a planar target, setting the timescale for excavation, the remaining coefficients $\alpha_{i j k}$ mediate the dependence of the local velocity of erosion on the surface morphology and incorporate the effect of beam parameters such as the average energy and flux, and those characterizing the statistics of energy deposition via collision cascades within the target. An important example is, of course, provided by those associated with Sigmund's Gaussian distribution [2, 11, 12], although improvements in the description of collision cascades (see, e.g., [23] for the case of $\mathrm{Cu}$ ) can be readily incorporated into equation (3). Thus for instance, using Sigmund's statistical description, the coefficients $\alpha_{2 j}$ are positive favoring excavation at surface minima rather than at surface maxima, leading to the ripple instability $[24,2]$. An opposing stabilizing effect that would provide negative contributions to $\alpha_{2 j}$ would be, for instance, direct knock-on effects [16], but we will neglect them since they are believed to be typically small for the range of energies of interest here [25].

As for the local rate of addition for mobile species back into the immobile target, naturally it should be susceptible to enhancement by the presence of the beam due to the induced increase in the density of diffusing species, but within our framework we would like to have also surface diffusion currents which are not necessarily proportional to the ion flux but are, rather, thermally activated. Such currents are known to favor, on average, addition at concave surface positions (minima) rather than at convex surface locations (maxima) [21, 22]. To this end, we allow for a non-zero thickness of mobile material $R_{\text {eq }}$ even in the absence of excavation $\left(\Gamma_{\mathrm{ex}}=0\right)$ or redeposition $(\phi=1)$ and write

$$
\Gamma_{\mathrm{ad}}=\gamma_{0}\left[R-R_{\mathrm{eq}}\left(1-\gamma_{2} \nabla^{2} h\right)\right],
$$

where $\gamma_{0}$ is the mean nucleation rate for a flat surface. This form for the rate of addition allows us to implement the GibbsThomson effect [21, 22]. Indeed, it can be shown [18] that, in the absence of erosion, equations (1), (2) and (4) reproduce exactly Mullins' thermal surface diffusion with $D=D_{\mathrm{s}}$ the surface diffusivity, $R_{\text {eq }}=v_{\mathrm{s}} / n_{v}$, where $v_{\mathrm{s}}$ is the concentration of mobile surface species, and $n_{v}^{-1}$ the atomic volume $\gamma_{2}=$ $\gamma /\left(k_{\mathrm{B}} T n_{v}\right) \geqslant 0$, with $\gamma$ the surface free energy per unit area (which we will assume isotropic) and $k_{\mathrm{B}}$ Boltzmann's constant. Note, generalizations of this formula are feasible, in which, for

5 In such a case we assume the projection of the beam to be along the positive $x$ direction. example, the surface tension $\gamma$ is anisotropic but, being here mostly interested in the case of amorphizable targets, we will mostly consider isotropic surface tension.

\section{Normal incidence}

\subsection{Effective equation}

We start by considering the simplest case, in which ions impinge along trajectories that are perpendicular to the initial target plane. In such a case, the shape of the excavation rate simplifies, since coefficients multiplying terms that break the $x \leftrightarrow-x$ symmetry are zero and the $x \leftrightarrow y$ symmetry is restored. This can be explicitly checked whenever analytic expressions for coefficients $\alpha_{i j k}$ are available as functions of the incidence angle, as in the case of Sigmund's Gaussian distribution $[11,12]$ or the modified exponential distribution in [23]. Thus, $\Gamma_{\mathrm{ex}}$ (equation (3)) simplifies to

$$
\Gamma_{\mathrm{ex}}=\alpha_{0}\left[1+\alpha_{2} \nabla^{2} h+\alpha_{4} \nabla^{4} h+\alpha_{6}(\nabla h)^{2}\right] .
$$

In order to gain insight into the surface dynamics that is described by this model, we can perform a numerical integration of a simplified 1D surface that is obtained if we set to zero all space derivatives in the $y$ coordinate. Results are shown in figure 1. As time proceeds, we can appreciate in panel (a) the appearance of a typical cell (dot) size that increases with time. Moreover, the thickness $R$ of the mobile layer evolves rapidly and adapts to the shape determined by the $h$ field. Note, we have employed a value $\epsilon \equiv \alpha_{0} /\left(\gamma_{0} R_{\text {eq }}\right)$ that is small in standard experimental conditions [18]. The smallness of this parameter quantifies the large separation in timescales between mobile species diffusion and the much slower driving associated with the ion beam. Given that $R$ evolves with the fast (diffusion) processes ${ }^{6}$, this means one can approximately set $\partial_{t} R \approx 0$ at every instant and solve $R$ in terms of (the derivatives of) $h$ from equation (1). Mathematically, such a program can be consistently implemented by setting up a perturbation expansion in terms of $\epsilon$ and employing a multiple scale analysis. Details can be found in [18]. The final outcome is a closed evolution equation that only involves the height field. To lowest nonlinear order and in the present normal incidence conditions, the equation is

$$
\partial_{t} h=v_{0}+v \nabla^{2} h-\mathcal{K} \nabla^{4} h+\lambda^{(1)}(\nabla h)^{2}-\lambda^{(2)} \nabla^{2}(\nabla h)^{2},
$$

where

$$
\begin{gathered}
v_{0}=-\alpha_{0} \phi, \quad v=-\alpha_{0} \phi \alpha_{2}, \\
\mathcal{K}=D R_{\mathrm{eq}} \gamma_{2}+\alpha_{0}\left[\phi \alpha_{4}-\alpha_{2} \Delta\right], \\
\lambda^{(1)}=-\alpha_{0} \phi \alpha_{6}, \quad \lambda^{(2)}=-\alpha_{0} \alpha_{6} \Delta, \\
\Delta=\frac{\bar{\phi} D}{\gamma_{0}}-\phi R_{\mathrm{eq}} \gamma_{2} .
\end{gathered}
$$

Equation (6) generalizes the KS equation through appearance of the conserved nonlinearity with parameter

6 Indeed, $R$ evolves exponentially fast with rate $\gamma_{0}$, as is apparent when substituting (4) into equation (1). 

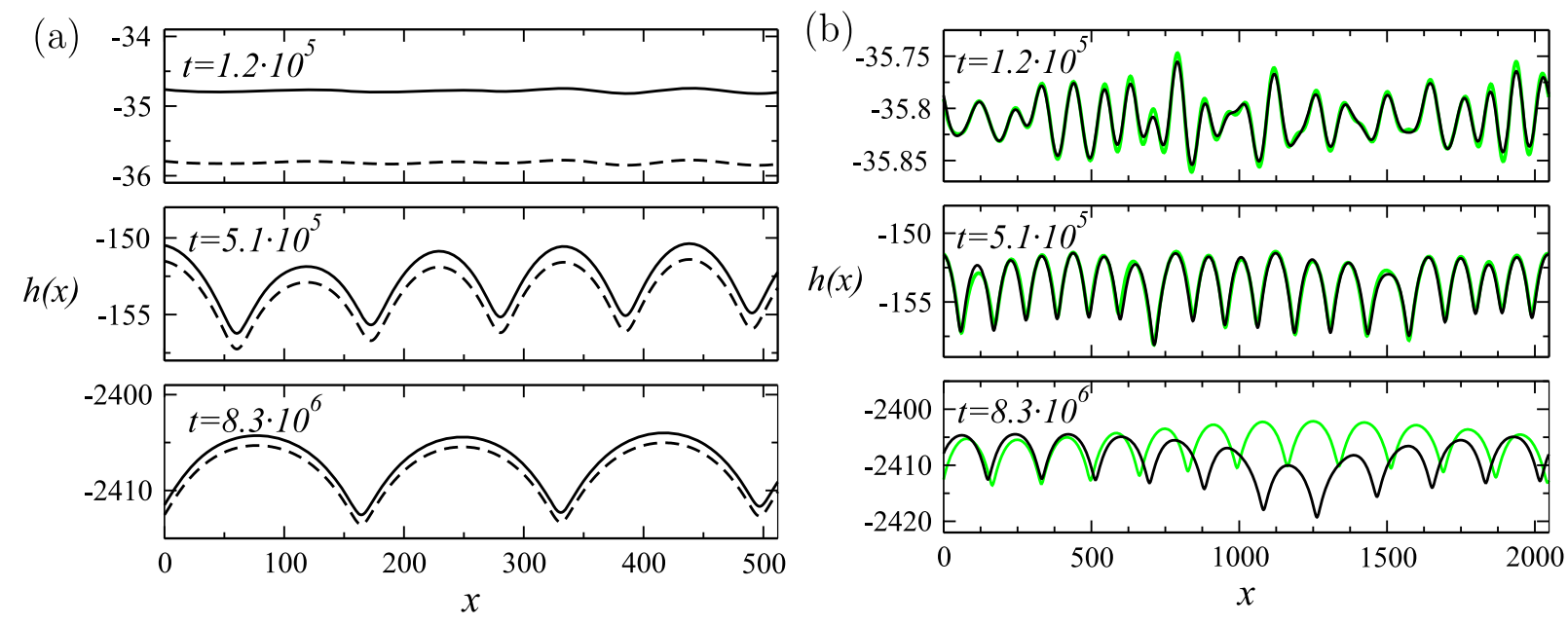

Figure 1. (a) Time evolution for the mobile surface layer $R$ (solid line) added to the surface height value $h$ (dashed line) for a 1D interface as described by equations (1)-(2) using (4) and (5) for $\bar{\phi}=0.99, \alpha_{0}=0.03, \alpha_{2}=30, \alpha_{6}=-3, \alpha_{4}=0, R_{\mathrm{eq}}=\gamma_{0}=\gamma_{2}=1$ and $D=10$. (b) Dynamics of $h$ described by the 1D two-field model for parameters as in (a) (green line) and by the effective equation (6) with parameters as obtained from (7): $v_{0}=-0.0003, v=-0.009, \mathcal{K}=1.099, \lambda^{(1)}=0.0009$ and $\lambda^{(2)}=0.8901$ (black line). In both panels time increases top to bottom. Notice the different vertical scales.

$\lambda^{(2)}[16]^{7}$. It has also appeared in different contexts, such as growth of amorphous thin films [27], and even in (macroscopic) pattern formation on snow fields [28]. In the case of IBS nanopatterning, it was initially obtained within a higher expansion of one-field models [29], although in such a case it is seen to break down as a continuum description [30, 31], see below. Soon after, it was properly obtained [16] within a two-field description of IBS based on a model with a different-albeit asymptotically equivalent $[32,18]$ —choice for the rate of addition $\Gamma_{\text {add }}$.

As mentioned above, for typical distributions of energy deposition, $\alpha_{2}$ is a positive number [2,12,23] and therefore $v<0$. Thus, just as in the KS system, equation (6) features a band of linearly unstable Fourier modes $h_{k}(t) \simeq \exp \left(\omega_{k} t\right)$, associated with $k$ values that make the dispersion relation $\omega_{k}=-v k^{2}-\mathcal{K} k^{4}$ a positive number. Generically, on the other hand, $\mathcal{K}$ is expected to be a positive coefficient. Thus, for short times during which nonlinearities are negligible, equation (6) predicts formation of a dot (cell) structure with a typical size provided by the inverse of the wavevector, $k^{\ell}$, that makes $\omega_{k}$ a positive maximum. A typical time evolution as predicted by (6) is shown in figure 1 for a simple 1D interface, and compared with the result of the full two-field model for the same parameters. Figure 1 also shows that, provided $\epsilon$ is small, both descriptions (two-field and effective one-field) yield very similar results for the morphology. This statement can be made more quantitative by comparing the time evolution of the roughness $W(t)$ (root mean square of the height fluctuations) and the dot wavelength $l(t)$ (measured as twice the average distance between consecutive minima and maxima) in figure 2 . We can distinguish roughly three different time regimes in the evolution. The first one (for $t \lesssim 3 \times 10^{5}$ units) is associated with the linear instability, corresponding to the selection of a time-independent wavelength and exponential growth of the

7 Termed as a conserved Kardar-Parisi-Zhang (CKPZ) nonlinear term in the kinetic roughening literature [26]. roughness. After that, an intermediate transient occurs (up to $t \lesssim 2 \times 10^{6}$ units) during which the roughness grows at a much lower rate and the pattern wavelength increases following an effective power law (coarsening) $l(t) \sim t^{0.45}$. For very long times (fluence) both the roughness and the wavelength reach stationary values. It is remarkable that both descriptions provide the same outcome, both qualitatively and quantitatively.

The behavior of the roughness and the wavelength from medium to long times is determined by the two nonlinearities that appear in equation (6). As is well known, the linearization of equation (6) predicts unbounded growth of the dot amplitude, so that the stationary state that is reached necessarily occurs from the interplay of the nonlinear terms. Before considering this, let us recall the fact that an equation such as equation (6) may under some circumstances also lead to unbounded amplitude growth. This is the case when socalled cancellation modes occur in the system. These are Fourier modes of the height that, while being linearly unstable, are associated with an exact cancellation of the nonlinear terms in the equation of motion. Thus, any unstable mode with wavevector magnitude $k_{0}$ is a cancellation mode if $\lambda^{(1)}+$ $k_{0}^{2} \lambda^{(2)}=0$. For this condition to occur, necessarily (within the above sign convention) $\lambda^{(1)}$ and $\lambda^{(2)}$ must have opposite signs. This is the case generically for one-field derivations [29-31], for whose parameter values equation (6) leads to exponential blow-up. However, from the formula above we see that equation (6) can be free of cancellation modes as long as $\lambda^{(1)} \lambda^{(2)}>0$. Even if $\lambda^{(1)} \lambda^{(2)}<0$ we will have bounded growth of the dot amplitude if $k_{0}$ is linearly stable, which is only verified if $k_{0}=\left|\lambda^{(1)} / \lambda^{(2)}\right|^{1 / 2}>|\nu / \mathcal{K}|^{1 / 2}$. Indeed $\left|\lambda^{(1)} / \lambda^{(2)}\right| \gg|\nu / \mathcal{K}| \sim \epsilon$ since $\left|\lambda^{(1)} / \lambda^{(2)}\right|=1 /|\Delta| \sim O(1)$ in our $\epsilon$ expansion, and therefore modes for which nonlinear terms cancel one another are outside the unstable band for small $\epsilon$ and decay exponentially in time. Thus, the two-field formulation allows us to eliminate issues of mathematical illposedness met by previous continuum models. 

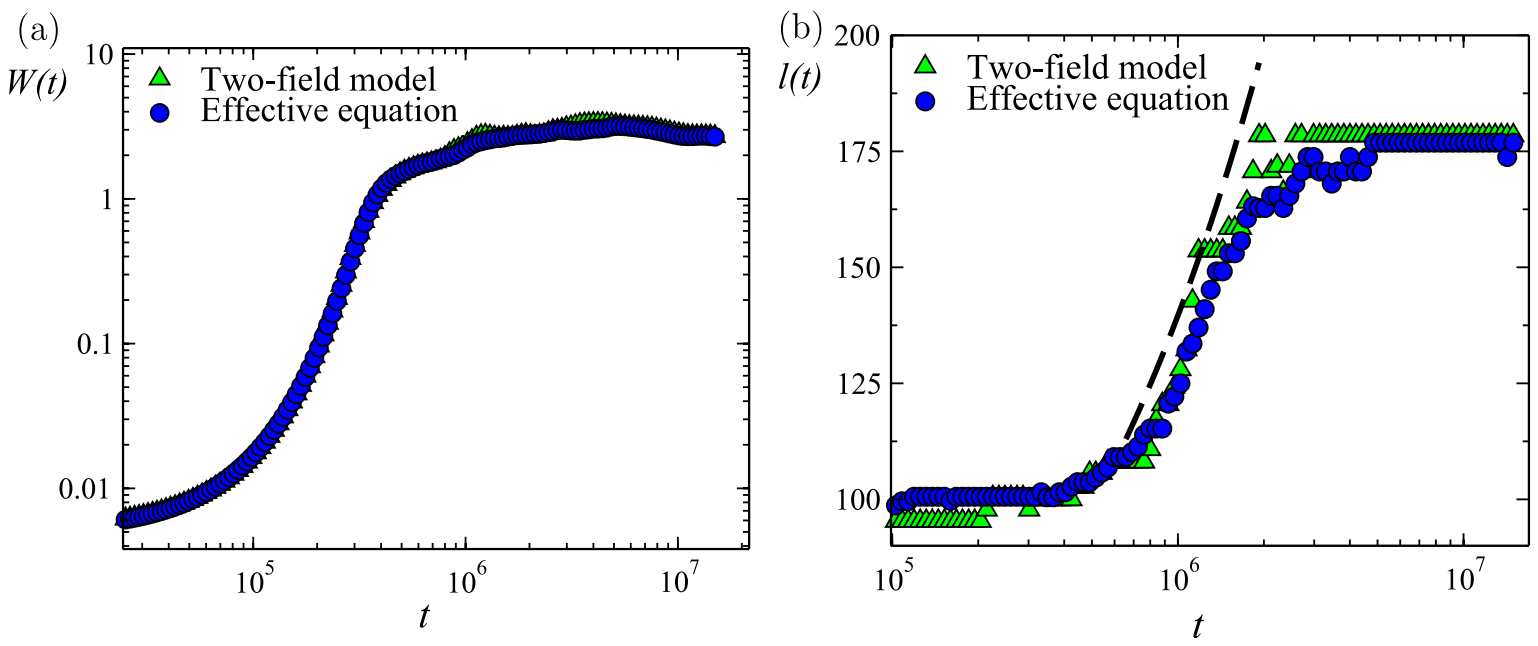

Figure 2. Temporal evolution of the roughness (a) and dot wavelength (b) for the (1D) two-field model (triangles) and for the (1D counterpart of the) effective equation (6) (circles) for the same parameters as in figure 1. The dashed line corresponds to a power law as $l \sim t^{0.45}$.

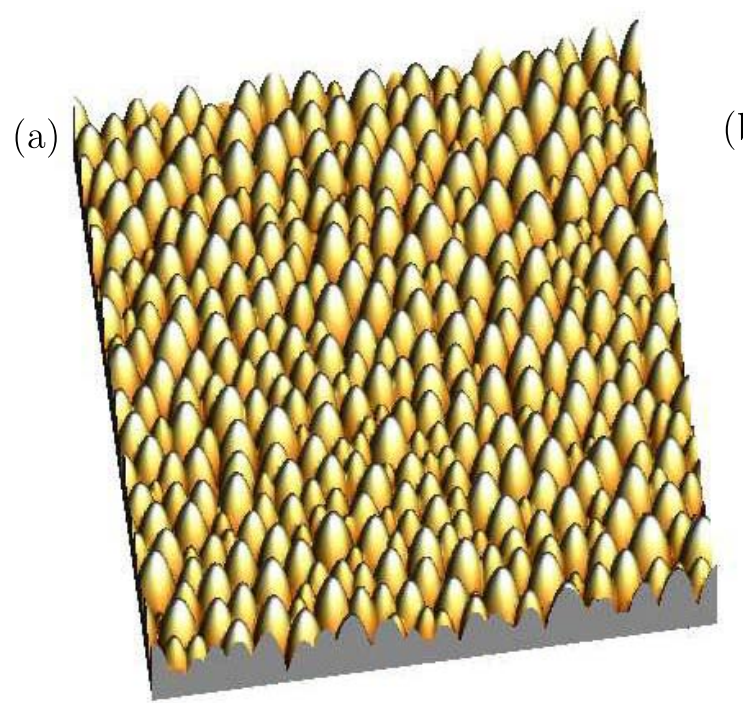

(b)

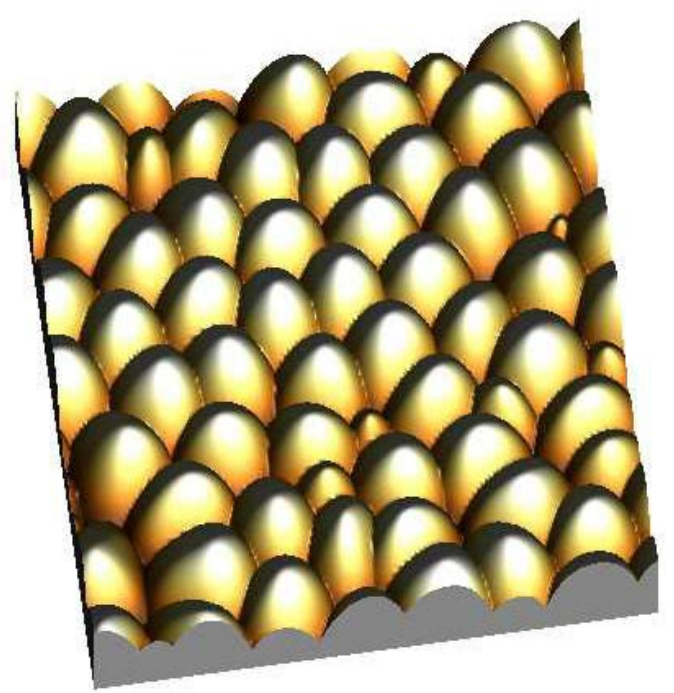

Figure 3. 3D top views of morphologies predicted by equation (9) for $r=50$ at (a) $t=50$ and (b) $t=955$.

\subsection{Temporal evolution in $2 D$}

A convenient way to fully explore the parameter space of the full $2 \mathrm{D}$ equation (6) is to rescale time, target and growth coordinates to a primed system $t=\left(\mathcal{K} / \nu^{2}\right) t^{\prime}, \mathbf{x}=(\mathcal{K} /|v|)^{1 / 2} \mathbf{x}^{\prime}$ and $h=\left(|\nu| / \lambda^{(1)}\right) h^{\prime}$. Thus, for negative values of $v$, equation (6) transforms into the equivalent single-parameter system (we drop the primes and the uniform velocity term $v_{0}$ ):

$$
\partial_{t} h=-\nabla^{2} h-\nabla^{4} h+(\nabla h)^{2}-r \nabla^{2}(\nabla h)^{2},
$$

where $r=\left(|v| \lambda^{(2)}\right) /\left(\mathcal{K} \lambda^{(1)}\right)$ is a ratio between crossover length scales associated with the linear and with the nonlinear terms. In this way, any combination of parameter values in the original equation can be studied in the equivalent system through the appropriate rescaling. Conversely, dependence of the system properties on $r$ can be translated into the original parameters once the coordinate rescaling is undone. Thus, for example, for the numerical studies in this section we have fixed the original equation parameters, equation (6), to $v_{0}=0$, $-v=\mathcal{K}=1$ and $\lambda^{(1)}=0.1$, varying $\lambda^{(2)}$ in order to check for the different values of $r$ and the different behaviors of equation (9).

Two snapshots of the time evolution of equation (9) are shown in figure 3 for $r=50$. We can appreciate the appearance of dots with a typical lateral size that coarsens with time. Figures 4 and 5 provide the height autocorrelation function, 2D height power spectral density (PSD) and the radial average of the latter, computed for the morphologies of figure 3. While the 2D and the radially averaged PSDs show coarsening (time shift to smaller wavevector values of the position of the main ring or peak) and long-wavelength disorder at long times, short-range order is made explicit by the bright 'rings' in the height autocorrelation function. As for the behavior of the roughness and wavelength (the latter now estimated from the distance to the origin of the first maxima of the autocorrelation function), they are computed in figure 6 
(a)

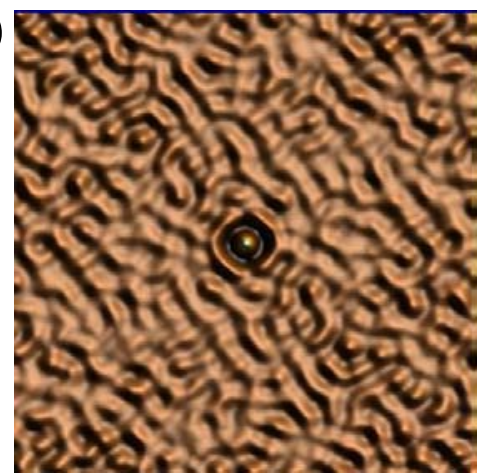

(b)

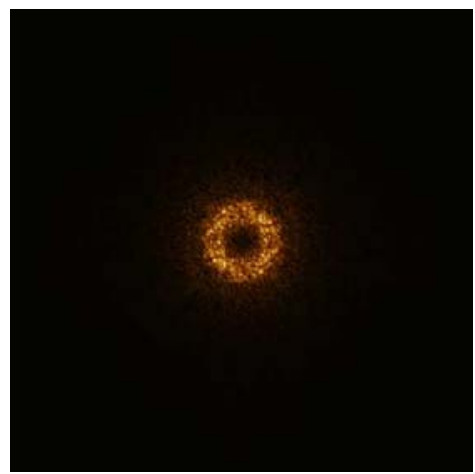

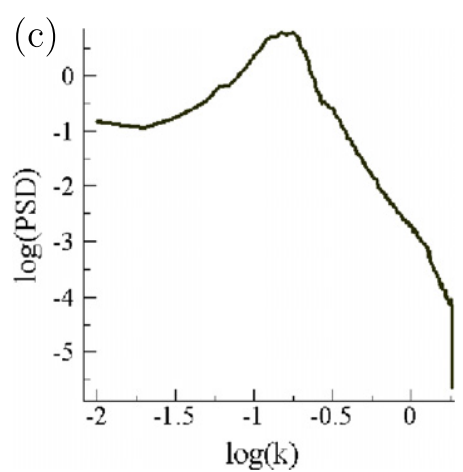

Figure 4. (a) Height autocorrelation function, (b) 2D PSD and (c) radially averaged PSD as computed for figure 3 (a).

(a)

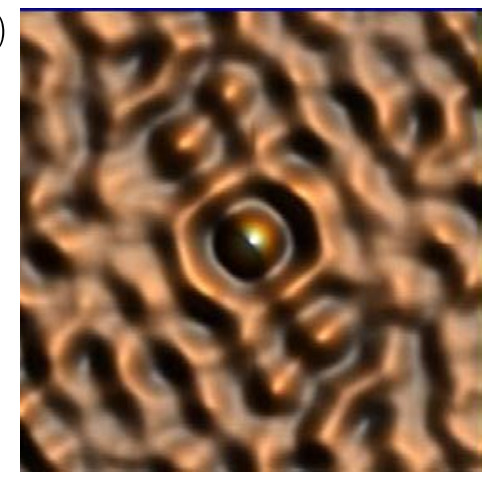

(b)

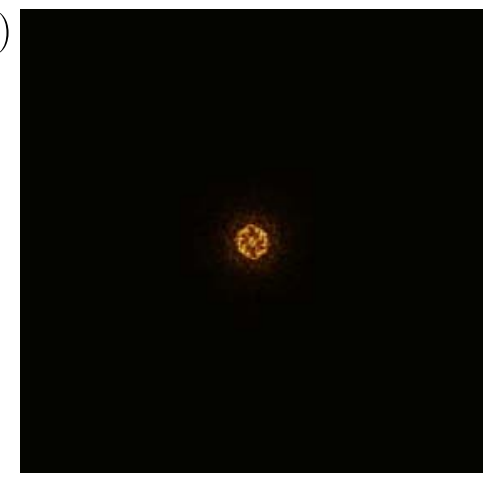

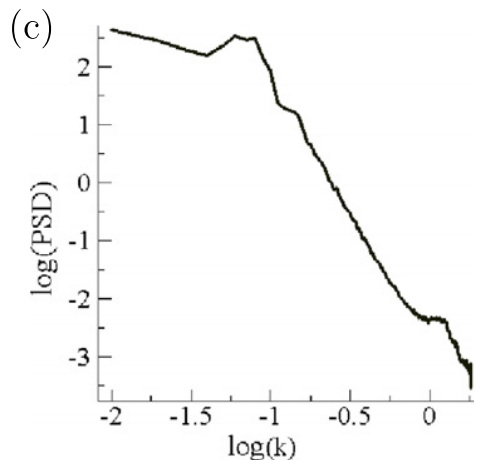

Figure 5. (a) Height autocorrelation function, (b) 2D PSD and (c) radially averaged PSD as computed for figure 3 (b).

(a)

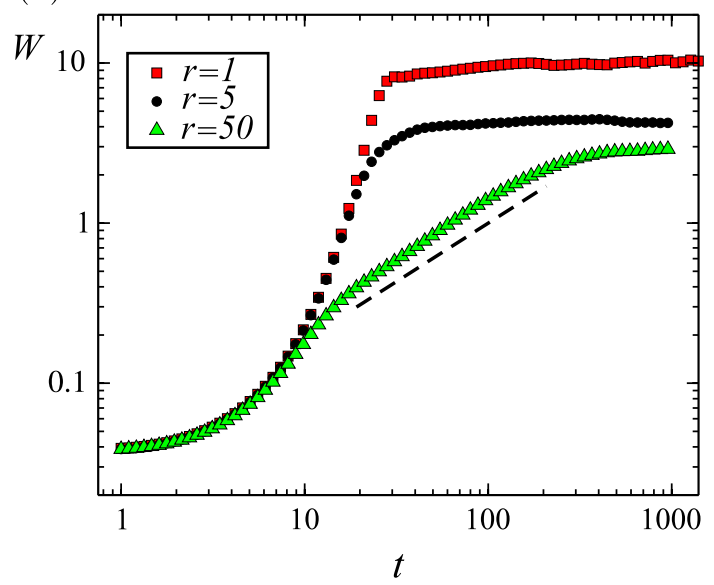

(b)

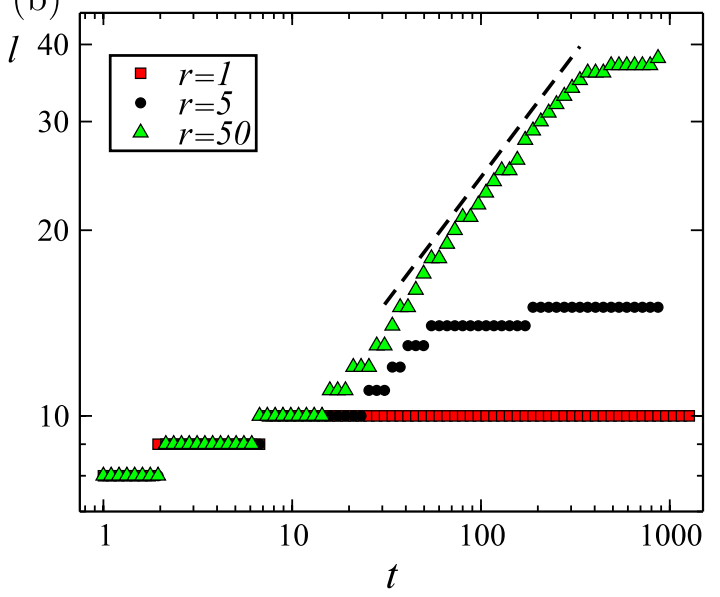

Figure 6. (a) Numerical values of the roughness and (b) dot size (wavelength) as predicted by equation (9) for different values of $r$. For reference, the dotted lines correspond to effective power law behavior as $W(t) \sim t^{0.73}$ and $l(t) \sim t^{0.40}$.

for several positive values of $r$. For increasing values of $r$, the stationary state is reached later and for decreasing saturated values of the roughness, while the duration of the intermediate time regime increases during which the roughness grows as an effective power law, e.g. $W(t) \sim t^{0.73}$ for $r=50$. We also see that, again for increasing $r$ values, the intermediate transient associated with wavelength coarsening lasts longer, e.g. the $l(t) \sim t^{0.40}$ behavior found for $r=50$. Actually, in the $r \rightarrow \infty$ limit equation (9) becomes the conserved KS equation, for which analytical and numerical estimates yield $W(t) \sim t$ and $l(t) \sim t^{1 / 2}$ [33-35]. Thus, we conclude in particular that the exponent values for finite positive $r$ are effective values depending on system parameters (summarized in $r$ ). This could explain the variety of exponents reported in the experimental literature: the value of the exponent is not universal but, rather, it is effectively and strongly dependent on transient behavior and/or finite size effects. 
(a)

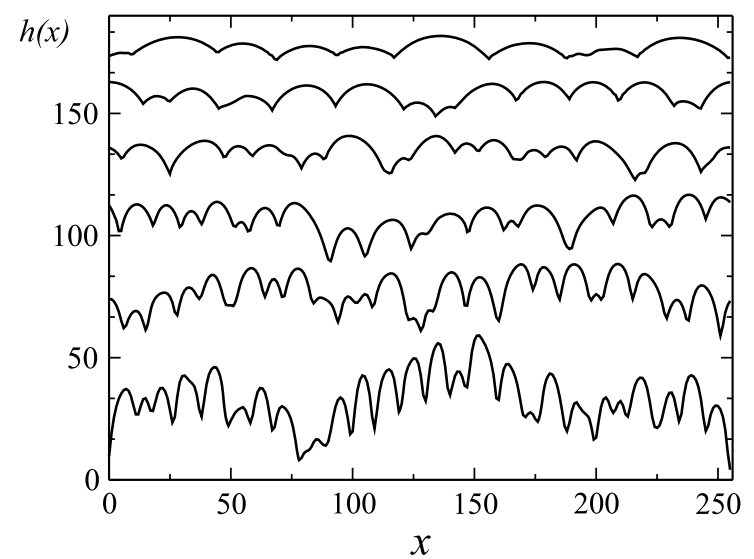

(b)

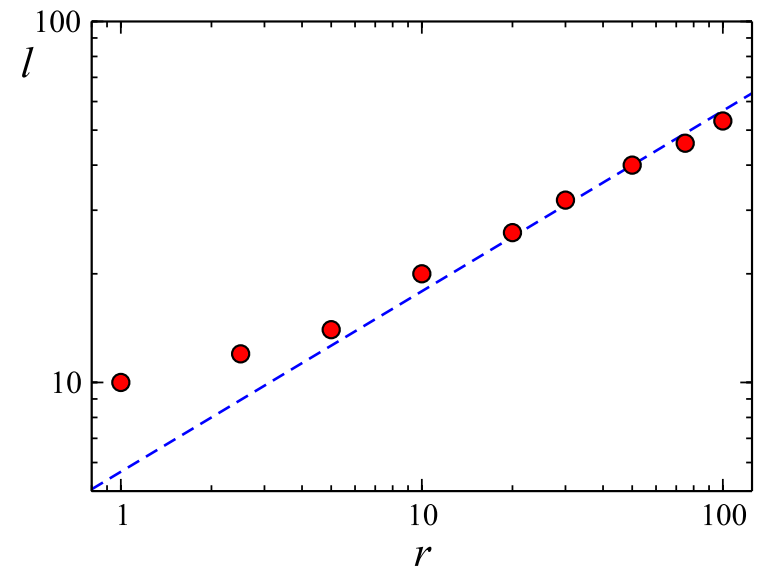

Figure 7. (a) Typical stationary transverse cut profiles predicted by equation (9) for (bottom to top) $r=1,2.5,5,10,20$ and 50 at $t=10000$. (b) Stationary dot average lateral size as functions of $r$ at $t=10000$. The dashed line in (b) corresponds to the analytical prediction given by equation (10).

\subsection{Stationary pattern properties}

In the presence of coarsening, which is generically expected for large positive values of $r$ in equation (6), the values of the dot amplitude and lateral size-rather than wavelength, since the pattern is no longer sinusoidal once nonlinear effects set indiffer from the linear estimates. Actually, there is no meaning to linear amplitude, since this quantity is nonstationary within the linear approximation, while the linear dot wavelength is $l^{\ell} \propto(\mathcal{K} /|\nu|)^{1 / 2}$. Typical stationary transverse cuts are depicted in figure 7(a) for different values of $r$. As observed here, larger stationary dot lateral sizes and less disordered profiles are achieved for increasing values of $r$.

We can use equation (9) in order to derive approximate predictions for the parameter dependence of the stationary amplitude and dot size. Thus, we make the observations that (i) coarsening is induced by the $\lambda^{(2)}$ nonlinearity, (ii) coarsening is interrupted by the $\lambda^{(1)}$ nonlinearity and (iii) the stationary shape of individual dots can be rather well approximated by inverted circular paraboloids of amplitude $A$ and radius $l / 2$, of the form $h(x, y)=A-\left(4 A / l^{2}\right)\left(x^{2}+y^{2}\right)$. In order to estimate the relation between $A, l$ and $r$ we assume that, when coarsening stops, the spatial averages of the two nonlinear terms can be equated within the lateral extent of a single dot. This leads to the condition

$$
\left\langle(\nabla h)^{2}\right\rangle_{\mathrm{dot}}=r\left\langle\nabla^{2}(\nabla h)^{2}\right\rangle_{\mathrm{dot}} \Rightarrow l=\sqrt{32 r},
$$

where $\langle\cdot\rangle_{\text {dot }}$ denotes space average over a single dot. Actually, this result is almost identical to an analogous one obtained for the 1D case [36]. Comparison to results from simulations can be found in figure 7(b). We can see that relation (10) holds rather accurately for relatively large $r$ values, for which coarsening is substantial and loses accuracy for smaller $r$ values. Moreover, from figure 7(a) we see that $A$ is essentially independent of $r$, except for small $r$ values for which we find a very disordered profile. Since we wish to extract the dependence of $A$ and $l$ on the original physical parameters we undo the rescaling, taking into account that lateral distances scale as $\mathbf{x}$ while vertical distances scale as $h$. In summary, we expect for moderately large $r$ values that

$$
\begin{gathered}
l \sim \sqrt{\frac{\mathcal{K}}{|\nu|} r}=\sqrt{\frac{\lambda^{(2)}}{\lambda^{(1)}}}=\sqrt{\frac{\bar{\phi} D}{\phi \gamma_{0}}-R_{\mathrm{eq}} \gamma_{2},} \\
A \sim \frac{|\nu|}{\lambda^{(1)}}=\frac{\alpha_{2}}{\alpha_{6}},
\end{gathered}
$$

where we have used equations (7) and (8). We see, on the one hand, that the stationary values of $l$ and $A$ depend purely on terms of erosive origin, such as $v$ and both $\lambda^{(j)}$ s. Nevertheless, while $A$ has no dependence on the transport processes, $l$ does depend on thermally related coefficients, such as $D$ and $R_{\text {eq }}$ can be. If we take for definiteness expressions for the $\alpha_{j}$ parameters stemming from Sigmund's Gaussian [12], and ignore the potential dependence of $D$ and $R_{\text {eq }}$ with the ion beam, we find that $A^{\text {Sigmund }} \sim a \sim E$, where $a$ is the average penetration depth and $E$ is the average ion energy, while $l^{\text {Sigmund }}$ is energy-independent, and that both $A^{\text {Sigmund }}$ and $l^{\text {Sigmund }}$ are flux-independent. To the best of our knowledge, these are the first analytical estimates of the stationary features of IBS nanopatterns; see below for a discussion in terms of experimental evidence.

\section{Oblique incidence for rotating targets}

There is a different way in which the loss of in-plane symmetry implied by an oblique beam can be circumvented, apart from the previous normal incidence condition. This is socalled Zalar rotation [37], by which the sample is rotated simultaneously with irradiation at oblique incidence, in order to suppress ripple formation while typically maximizing the sputtering yield. Indeed, led by the work in [7] for InP, in recent years this approach has been successfully employed in order to produce nanometric dots with a remarkable degree of uniformity and lateral order on a variety of targets, see a recent overview in [5]. 
Since we already have a closed evolution equation for the surface height under oblique incidence conditions [17, 18], it is natural to employ it in order to derive an equation for the present case of rotating targets. In this, we follow Bradley's approach to Zalar rotation within the context of one-field theories [38]. Thus, let us perform a rotation of angle $\varphi$ in the target coordinates to a new system with subindex ${ }_{r}$, as

$$
\begin{gathered}
x_{\mathrm{r}}=x \cos \varphi+y \sin \varphi, \\
y_{\mathrm{r}}=-x \sin \varphi+y \cos \varphi,
\end{gathered}
$$

so that space derivatives are related as

$$
\begin{gathered}
\frac{\partial}{\partial x}=\cos (\varphi) \frac{\partial}{\partial x_{\mathrm{r}}}+\sin (\varphi) \frac{\partial}{\partial y_{\mathrm{r}}} \\
\frac{\partial}{\partial y}=-\sin (\varphi) \frac{\partial}{\partial x_{\mathrm{r}}}+\cos (\varphi) \frac{\partial}{\partial y_{\mathrm{r}}}
\end{gathered}
$$

If we perform this change of coordinates in the height equation for oblique incidence $\theta \neq 0[17,18]$, we would get the equation of motion for a beam that is not aligned with the $x$ axis but, rather, at an oblique azimuthal angle $\varphi$. We now assume that the sample is rotating with a constant angular velocity, $\dot{\varphi}$, fast enough so that, effectively, it is as if bombardment were taking place at all possible azimuthal incidences simultaneously. In such a case we can get an effective evolution equation by averaging over all values $0 \leqslant$ $\varphi \leqslant 2 \pi$. The result is [32]

$$
\begin{aligned}
\partial_{t} h & =v_{\mathrm{r}} \nabla^{2} h-\mathcal{K}_{\mathrm{r}} \nabla^{4} h+\lambda_{\mathrm{r}}^{(1)}(\nabla h)^{2} \\
& -\lambda_{\mathrm{r}}^{(2)} \nabla^{2}(\nabla h)^{2}-\lambda_{\mathrm{r}}^{(3)} \nabla \cdot\left[\left(\nabla^{2} h\right) \nabla h\right],
\end{aligned}
$$

where the coefficients

$$
\begin{gathered}
v_{\mathrm{r}}=\frac{v_{x}+v_{y}}{2}, \quad \mathcal{K}_{\mathrm{r}}=\frac{3 \mathcal{K}_{x, x}+3 \mathcal{K}_{y, y}+\mathcal{K}_{x, y}+\mathcal{K}_{y, x}}{8}, \\
\lambda_{\mathrm{r}}^{(1)}=\frac{\lambda_{x}^{(1)}+\lambda_{y}^{(1)}}{2}, \quad \lambda_{\mathrm{r}}^{(2)}=\frac{\lambda_{x, x}^{(2)}+\lambda_{y, y}^{(2)}+\lambda_{x, y}^{(2)}+\lambda_{y, x}^{(2)}}{4}, \\
\lambda_{\mathrm{r}}^{(3)}=\frac{\lambda_{x, x}^{(2)}+\lambda_{y, y}^{(2)}-\lambda_{x, y}^{(2)}-\lambda_{y, x}^{(2)}}{4}
\end{gathered}
$$

are expressed in terms of those of equation (42) in [18]. As expected, linear and nonlinear terms that contain odd derivatives in space coordinates do not contribute after angular average (the mathematical statement of the restoration of inplane symmetry) and the system is actually isotropic in the target coordinates. However, note that this does not mean that the equation is equal to the one for normal incidence. Remarkably, in principle now the additional term appears $\nabla$. $\left[\left(\nabla^{2} h\right) \nabla h\right]$, which is naturally of the same order in powers of $h$ and in space derivatives as $\nabla^{2}(\nabla h)^{2}$. Both terms are actually equivalent in the $1 \mathrm{D}$ case. In the 2D case, one can show [32] that the only other possible isotropic terms of the same order in $h$ and space derivatives are $\left(\nabla^{2} h\right)^{2}$ and $(\nabla h) \cdot \nabla\left(\nabla^{2} h\right)$. Given that $\nabla \cdot\left[\left(\nabla^{2} h\right) \nabla h\right]=\left(\nabla^{2} h\right)^{2}+(\nabla h) \cdot \nabla\left(\nabla^{2} h\right)$, we see that the term with coefficient $\lambda_{\mathrm{r}}^{(3)}$ is a mere linear combination of the two.

We can make further progress by making use of the form of the coefficients (16) given by equation (43) in [18]. After substitution, we get

$$
\begin{aligned}
v_{\mathrm{r}}= & -\phi \alpha_{0}\left(\alpha_{2 x}+\alpha_{2 y}\right) / 2, \\
\mathcal{K}_{\mathrm{r}}= & D R_{\mathrm{eq}}\left(\gamma_{2 x}+\gamma_{2 y}\right) / 2 \\
& +\alpha_{0}\left(3 \alpha_{4 x x}+3 \alpha_{4 y y}+\alpha_{4 x y}+\alpha_{4 y x}\right) / 8 \\
& -\alpha_{0}\left(3 \Delta_{x} \alpha_{2 x}+3 \Delta_{y} \alpha_{2 y}+\Delta_{x} \alpha_{2 y}+\Delta_{y} \alpha_{2 x}\right) / 8, \\
\lambda_{\mathrm{r}}^{(1)}= & -\phi \alpha_{0}\left(\alpha_{6 x}+\alpha_{6 y}\right) / 2 \\
\lambda_{\mathrm{r}}^{(2)}= & -\phi \alpha_{0}\left(\Delta_{x} \alpha_{6 x}+\Delta_{y} \alpha_{6 y}+\Delta_{x} \alpha_{6 y}+\Delta_{y} \alpha_{6 x}\right) / 4, \\
\lambda_{\mathrm{r}}^{(3)} & =-\phi \alpha_{0}\left(\Delta_{x} \alpha_{6 x}+\Delta_{y} \alpha_{6 y}-\Delta_{x} \alpha_{6 y}-\Delta_{y} \alpha_{6 x}\right) / 4,
\end{aligned}
$$

where we have generalized the parameter $\Delta$ in (8) to $\Delta_{x, y}=$ $\bar{\phi} R_{\mathrm{eq}} \gamma_{0}^{-1}-\phi R_{\mathrm{eq}} \gamma_{2 x, y}$ in order to allow for the possibility of anisotropic surface tension coefficients, $\gamma_{2 x} \neq \gamma_{2 y}$. Finally, for the simpler (and more natural, for amorphous targets) case of isotropic surface tension on the target, $\gamma_{2 x}=\gamma_{2 y}=\gamma_{2}$, we have that $\Delta_{x}=\Delta_{y}=\Delta$, and the previous formulae simplify into

$$
\begin{aligned}
v_{\mathrm{r}}= & -\phi \alpha_{0}\left(\alpha_{2 x}+\alpha_{2 y}\right) / 2, \\
\mathcal{K}_{\mathrm{r}}= & D R_{\mathrm{eq}} \gamma_{2}+\alpha_{0}\left(3 \alpha_{4 x x}+3 \alpha_{4 y y}+\alpha_{4 x y}+\alpha_{4 y x}\right) / 8 \\
& -\alpha_{0} \Delta\left(\alpha_{2 x}+\alpha_{2 y}\right) / 2, \\
\lambda_{\mathrm{r}}^{(1)}= & -\phi \alpha_{0}\left(\alpha_{6 x}+\alpha_{6 y}\right) / 2, \\
\lambda_{\mathrm{r}}^{(2)} & =-\phi \alpha_{0} \Delta\left(\alpha_{6 x}+\alpha_{6 y}\right) / 2, \\
\lambda_{\mathrm{r}}^{(3)} & =0 .
\end{aligned}
$$

Remarkably, in the latter case the effective equation for rotating targets takes the exact same shape (albeit with different coefficients) as for the normal incidence case, i.e. equation (6). This provides an analytical justification for the strong similarities found for IBS nanopatterning onto rotating targets and under normal incidence conditions, generalizing analogous results obtained for one-field approaches [38, 39].

As in the case of normal incidence, for the sake of definiteness it is interesting to discuss equations (18) in the light of some specific assumption on the distribution of energy deposition. Similarly to the previous section, here we make the standard choice of Sigmund's Gaussian. Note that (18) predicts the coefficients $v_{\mathrm{r}}$ and $\lambda_{\mathrm{r}}^{(1)}$ to be essentially the means of their counterparts in the corresponding onefield equation (for oblique incidence) [11, 12]. Moreover, the coefficient $\lambda_{\mathrm{r}}^{(2)}$ is directly proportional to $\lambda_{\mathrm{r}}^{(1)}$. As is well known, Sigmund's description successfully predicts [2] the experimentally observed existence of a critical incidence angle $\theta_{\mathrm{c}}$ at which the ripples change their orientation by $90^{\circ}$. At this critical angle $\alpha_{2 x}=\alpha_{2 y}$, while for still larger angles $\alpha_{2 x}$ changes sign, $\alpha_{2 y}$ still being positive. Actually, for such a distribution of energy deposition $v_{\mathrm{r}}$ has been shown $[39,40]$ to become positive for still larger incidence angles, thus predicting the absence of pattern formation under such experimental conditions.

Finally, we can again employ the predictions of section 3 for the stationary dot size and amplitude in the case of large $r$, and adapt them to the present case of rotating targets. Thus, by 
(a)

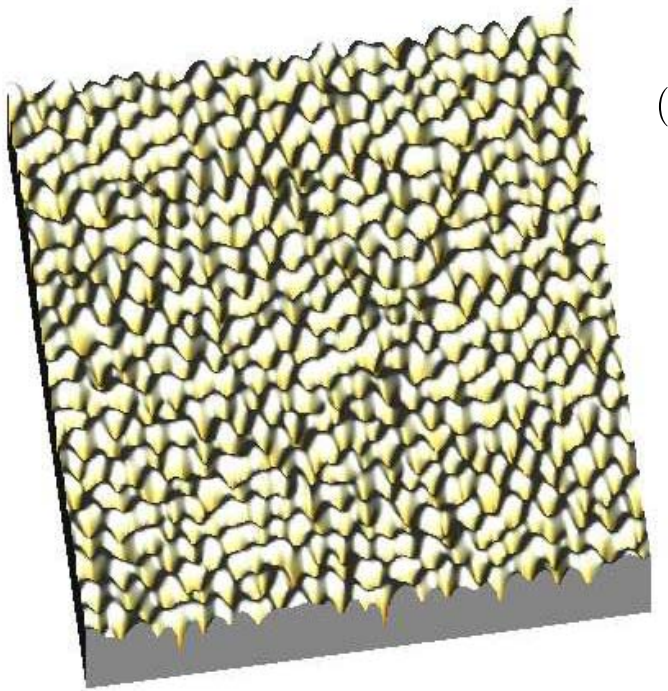

(b)

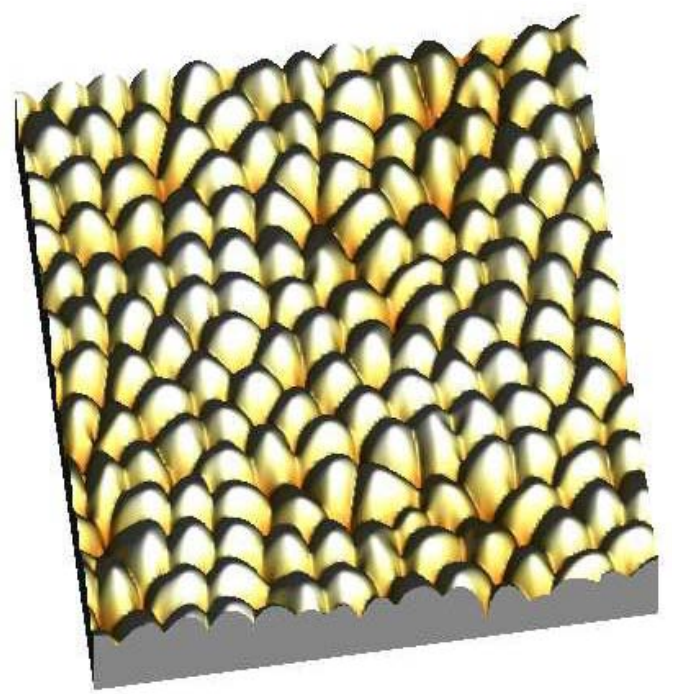

Figure 8. 3D top views of morphologies predicted by equation (15) for $v_{\mathrm{r}}=-1, \mathcal{K}_{\mathrm{r}}=1, \lambda_{\mathrm{r}}^{(1)}=0.1, \lambda_{\mathrm{r}}^{(2)}=0.5$ and $\lambda_{\mathrm{r}}^{(3)}=2$ at times (a) $t=50$ and (b) $t=955$.

(a)

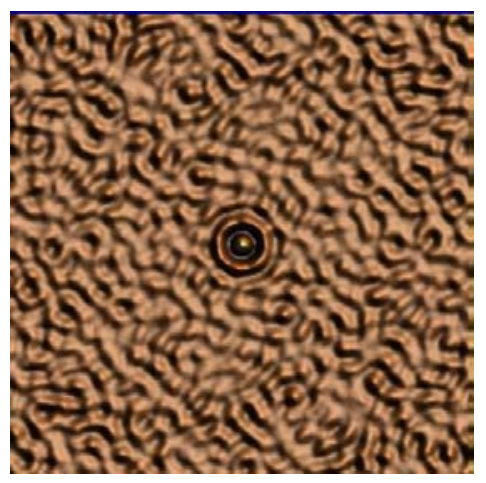

(b)

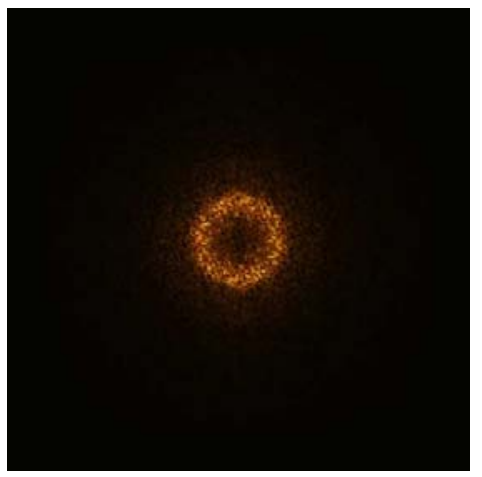

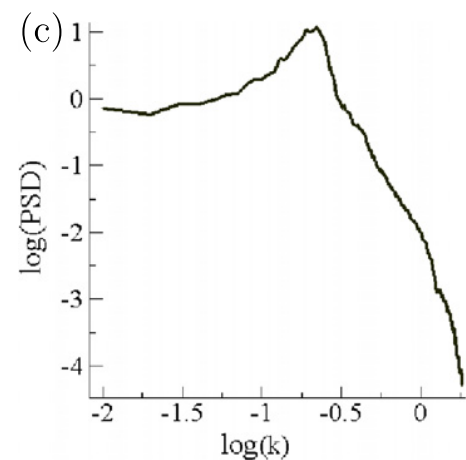

Figure 9. Height autocorrelation function (a), 2D PSD (b) and radially averaged PSD (c) as computed for figure 8 (a).

combining equations (11), (12) and (18), we get

$$
\begin{gathered}
l_{\mathrm{r}} \sim \sqrt{\frac{\lambda_{\mathrm{r}}^{(2)}}{\lambda_{\mathrm{r}}^{(1)}}}=\sqrt{\frac{\bar{\phi} D}{\phi \gamma_{0}}-R_{\mathrm{eq}} \gamma_{2}}, \\
A_{\mathrm{r}} \sim \frac{\nu_{\mathrm{r}}}{\lambda_{\mathrm{r}}^{(1)}}=\frac{\alpha_{2 x}+\alpha_{2 y}}{\alpha_{6 x}+\alpha_{6 y}},
\end{gathered}
$$

again very similar to those found for normal incidence. If we further assume Sigmund's energy deposition and that material transport is not affected by irradiation, one can readily check [12] that both $\alpha_{2 x, y}$ scale in the same way with average ion energy and flux, and similarly for $\alpha_{6 x, y}$. We thus get again $A_{\mathrm{r}}^{\text {Sigmund }} \sim a \sim E$, with no flux dependence, while $l_{\mathrm{r}}^{\text {Sigmund }}$ is again both energy- and flux-independent.

In the case of anisotropic surface tension on the target $\left(\gamma_{2 x} \neq \gamma_{2 y}\right)$, as expected for substrates that remain crystalline under irradiation [9], equation (15) does not reduce to equation (6) and we must take into account the term corresponding to $\lambda_{\mathrm{r}}^{(3)}$. In figure 8 we show two representative morphologies described by equation (15) at different times. Again, a dot structure appears at short times, which grows in amplitude and coarsens in wavelength (lateral size) for later times (see figures 9 and 10, where the height autocorrelation function, the 2D PSD, and the radially averaged PSD are depicted at two different times). In contrast to the normal incidence morphologies, in this case irregularly inclined paraboloids appear. For increasing values of $\lambda_{r}^{(3)}$, the stationary state is reached later, increasing the final wavelength (and the coarsening transient) and the height disorder of the pattern, as can be observed in figure 11 where the time evolution of the roughness and the wavelength are depicted for different values of $\lambda_{\mathrm{r}}^{(3)}$.

\section{Comparison to experiments and discussion}

Among the predictions of the present two-field description of IBS nanopatterning, we can make a distinction between those that depend sensitively on the details of the assumed specific distribution of energy deposition, and those that are robust to variations in such details. The latter properties include the formation of nanodots that usually coarsen with time and reach a stationary state characterized by a varying degree of short-range order and long-wavelength disorder. 
(a)

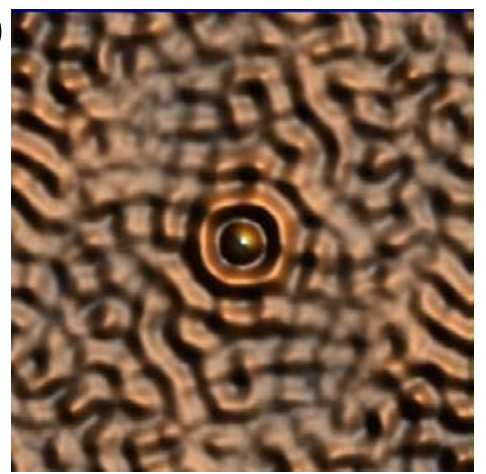

(b)

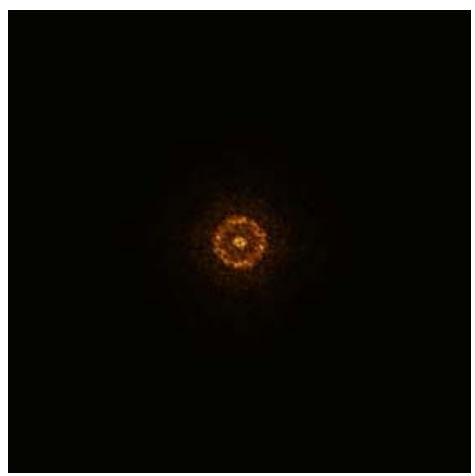

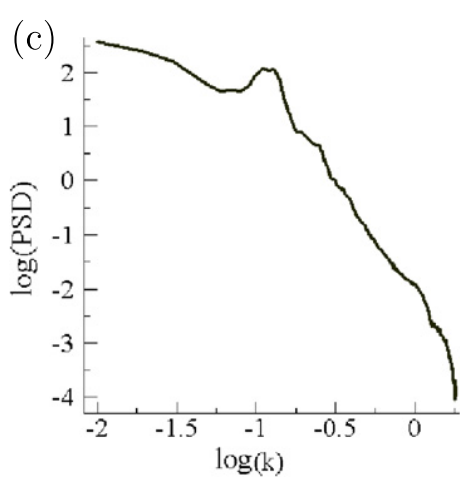

Figure 10. (a) Height autocorrelation function, (b) 2D PSD and (c) radially averaged PSD as computed for figure 8 (b).

(a)

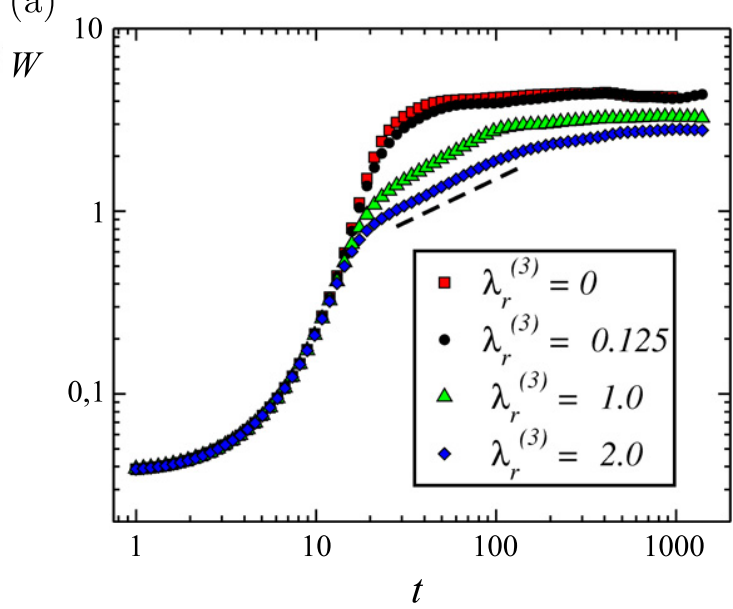

(b)

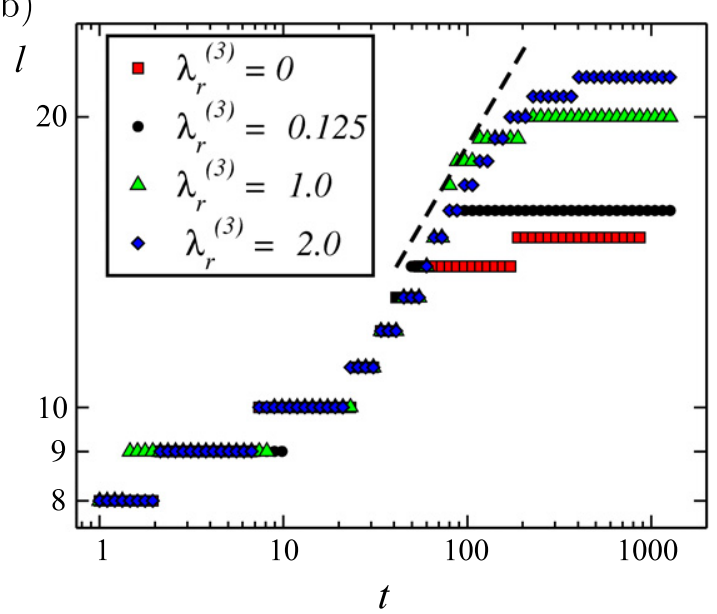

Figure 11. (a) Numerical values of the roughness and (b) dot size (wavelength) as predicted by equation (15) for $-v_{\mathrm{r}}=\mathcal{K}_{\mathrm{r}}=\lambda_{\mathrm{r}}^{(1)}=1$, $\lambda_{\mathrm{r}}^{(2)}=0.5$ and different values of $\lambda_{\mathrm{r}}^{(3)}$. For reference, the dotted lines correspond to effective power law behavior as $W(t) \sim t^{0.47}$ and $l(t) \sim t^{0.33}$.

Moreover, it is expected that, independently of the statistics of energy deposition, normal incidence conditions lead to similar patterns as obtained for rotating targets. All these features have indeed been found in experiments, as has been recently reviewed in [5]. This robustness lays moreover on deeper physical grounds such as symmetry, which explains its apparent ubiquity in different theoretical formulations of IBS [41].

There are still additional generic features that we expect to be sufficiently robust. Thus, equations (6)-(8) predict that, for conditions in which surface diffusion is essentially of thermal origin, the only parameter in (6) that is independent of the sputtering rate is $\mathcal{K}$. In such a case, an increase in the sputtering rate leads to faster saturation and a smaller stationary value of the dot size and degree of short-range order [32]. Indeed, the same trend has been found in IBS of Si targets at normal incidence [42] when comparing the morphologies obtained for an initial (100) surface orientation, and those obtained for the (111) orientation on which the sputtering rate is measured to be larger by $10 \%$. In the case that material transport is enhanced by irradiation, we expect $\mathcal{K}$ to depend on the sputtering rate. For a linear dependence, all coefficients in (6) are proportional to the flux and increasing flux rates will only induce a faster dynamics but the same stationary morphological features. We also expect that, since the inclusion of a non-zero $\lambda^{(3)}$ for targets with anisotropic surface tension yields dots with different shapes as compared with those obtained on isotropic substrates, this points towards surface tension effects in the specific shapes of the obtained nanostructures.

A second set of predictions from our present two-field description requires making specific assumptions on the shape of the statistics of energy deposition through collision cascades and on parameters related with material transport. An example was provided by the dependence of the stationary values of the dot amplitude and lateral size $A$ and $l$ on physical parameters for large values of $r$. In such a case, if we consider Sigmund's Gaussian distribution and ion-beam-independent transport of material, we have seen that the prediction is an energy-independent (respectively, linearly dependent) stationary dot wavelength (amplitude) and a flux-independent behavior of these magnitudes, both at normal incidence and for rotating targets. The dependence of the stationary ripple wavelength with the ion flux has been reported in a number of experiments. Thus, for instance, it has been found to be fluxindependent both for GaSb [43] and for InP [40], as predicted by (11) and (19), respectively. Thus partial agreement with 
experiments is achieved by the two-field implementation of Sigmund's energy distribution. On the other hand, these predictions for large values of $r$ contradict other observations on, for example, GaSb at normal incidence [43] or on $\mathrm{Si}$ onto rotating substrates [44] which also present other features, such as lack of coarsening and of long-wavelength kinetic roughening, which, interestingly, are generically present for small $r$. Thus, in order to assess the actual predictive power of this model, an unambiguous analytical formulation for the dependence of all the terms with the experimental parameters is still missing.

These results are mainly valid in the case of isotropic experiments. For cases in which such a symmetry is broken, either by the ion incidence angle or by preferential directions in the substrate (such as in the case of metals where no surface amorphization, and anisotropic surface tension and diffusion are expected), anisotropic patterns are predicted (see [18] for an extension to the case of general anisotropic experiments). Even in these cases, the isotropic symmetry can be restored by simultaneous target rotation, as discussed in the previous section. Although the resulting equation, equation (15), has not been extensively studied, anisotropic coefficients may give rise to new morphologies and may even change the sign of nonlinear terms (see equation (17)), breaking the up-down symmetry and giving rise to holes or craters instead of dots or mounds.

\section{Conclusion and outlook}

In this paper we have explored the two-field description of nanopattern formation by IBS under normal incidence conditions and for oblique incidence onto rotating targets. To some extent, the main virtues of such a continuum formulation over previous ones are: (i) the inclusion of local redeposition of eroded material, which allows us to couple in a natural (rather than ad hoc) way surface material transport with the dynamics of the surface itself. This has allowed us to account for important features of these patterns, such as shortrange in-plane ordering and wavelength coarsening, beyond curing mathematical issues on well-posedness that affected previous one-field models. (ii) Its generic nature that can be adapted to improvements in the description of the statistics of energy distribution, through modifications of the 'geometric' parameters $\alpha_{i j k}$ of the excavation rate $\Gamma_{\mathrm{ex}}$, and of material transport, through the dependence of $D$ and $R_{\text {eq }}$ on the ion beam [45]. Thus, for instance, we have seen how our twofield formulation allows us to analyze critically Sigmund's theory and transport material mechanisms and help to gain more insight on the processes involved in ion-beam sputtering. Moreover, appropriate changes in the parameters entering $\Gamma_{\mathrm{ex}}$ and $\Gamma_{\mathrm{ad}}$ would also allow for the inclusion of additional relaxation mechanisms that have been invoked elsewhere, such as direct knock-on and/or ballistic drift [46].

Under certain assumptions we have been able to extract for the first time analytical predictions for the values of the stationary dot amplitude and lateral size which are qualitatively very similar for the two geometries of incidence that have been studied, and seen that agreement with experimental observations is only partial. This fact, together with the lack of sufficient understanding of issues such as the radiation influence on surface material transport, and experimental results such as long-range order [47], lack of pattern formation at small incidence angles for some targets [48] and the role of preferential sputtering in the case of compound semiconductor targets [49], suggest the need for further microscopic work that improves our current parameterization of the excavation and addition rates, and thus allows for a wider predictive power of continuum theories of IBS nanopatterning.

\section{Acknowledgments}

We thank R Gago and L Vázquez for discussions. This work has been partially supported by MEC (Spain) through grant nos. FIS2006-12253-C06-01 and FIS2006-12253-C06-06, by CAM (Spain) through grant no. S-0505/ESP-0158 and by JCCM (Spain) through grant no. PAC-05-005.

\section{References}

[1] Carter G 2001 J. Phys. D: Appl. Phys. 34 R1

[2] Bradley R M and Harper J M E 1988 J. Vac. Sci. Technol. A 62390

[3] Sigmund P 1969 Phys. Rev. 184383

[4] Chan W L and Chason E 2007 J. Appl. Phys. 101121301

[5] Muñoz-García J et al 2007 Towards Functional Nanomaterials (Springer Series: Lecture Notes on Nanoscale Science and Technology) (New York: Springer) (Self-Organized Surface Nanopatterning by Ion Beam Sputtering) at press (arXiv:0706.2625v1)

[6] Facsko S et al 1999 Science 2851551

[7] Frost F, Schindler A and Bigl F 2000 Phys. Rev. Lett. 854116

[8] Gago R et al 2001 Appl. Phys. Lett. 783316

[9] Valbusa U, Boragno C and de Mongeot F B 2002 J. Phys.: Condens. Matter 148153

[10] Kuramoto Y 1984 Chemical Osillation, Waves and Turbulence (Heidelberg: Springer)

[11] Cuerno R and Barabási A-L 1995 Phys. Rev. Lett. 744746

[12] Makeev M, Cuerno R and Barabási A-L 2002 Nucl. Instrum. Methods Phys. Res. B 197185

[13] Kustner M, Eckstein W, Dose V and Roth J 1998 Nucl. Instrum. Methods Phys. Res. B 145320

[14] Aste T and Valbusa U 2004 Physica A 332548

[15] Aste T and Valbusa U 2005 New J. Phys. 7122

[16] Castro M, Cuerno R, Vázquez L and Gago R 2005 Phys. Rev. Lett. 94016102

[17] Muñoz-García J, Castro M and Cuerno R 2006 Phys. Rev. Lett. 96086101

[18] Muñoz-García J, Cuerno R and Castro M 2008 Phys. Rev. B 78205408

[19] Umbach C C, Headrick R L and Chan K-C 2001 Phys. Rev. Lett. 87246104

[20] Tiedje T and Ballestad A 2008 Thin Solid Films 516 3705-28

[21] Mullins W W 1957 J. Appl. Phys. 28333

[22] Mullins W W 1959 J. Appl. Phys. 3077

[23] Feix M et al 2005 Phys. Rev. B 71125407

[24] Sigmund P 1973 J. Mater. Sci. 81545

[25] Sigmund P 1981 Sputtering by Ion Bombardment. Theoretical Concepts ed R Behrisch (Berlin: Springer)

[26] Barabási A-L and Stanley H E 1995 Fractal Concepts in Surface Growth (Cambridge: Cambridge University Press)

[27] Raible M et al 2000 Europhys. Lett. 5061

[28] Tiedje T et al 2006 J. Geophys. Res. 111 F02015

[29] Kim T C et al 2004 Phys. Rev. Lett. 92246104 
[30] Castro M and Cuerno R 2005 Phys. Rev. Lett. 94139601

[31] Kim T C et al 2005 Phys. Rev. Lett. 94139602

[32] Muñoz-García J 2007 PhD Thesis Universidad Carlos III de Madrid

[33] Raible M, Linz S J and Hänggi P 2000 Phys. Rev. E 621691

[34] Frisch T and Verga A 2006 Phys. Rev. Lett. 96166104

[35] Politi P and Misbah C 2006 Phys. Rev. E 73036133

[36] Muñoz-García J, Cuerno R and Castro M 2006 Phys. Rev. E 74 R050103

[37] Zalar A 1985 Thin Solid Films 124223

[38] Bradley R M 1996 Phys. Rev. E 546149

[39] Frost F 2002 Appl. Phys. A 74131

[40] Frost F and Rauschenbach B 2003 Appl. Phys. A 771
[41] Castro M et al 2007 New J. Phys. 9102

[42] Gago R et al 2006 Appl. Phys. Lett. 89233101

[43] Facsko S, Kurz H and Dekorsy T 2001 Phys. Rev. B 63165329

[44] Ziberi B, Frost F and Rauschenbach B 2006 Appl. Phys. Lett. 88173115

[45] Ditchfield R and Seebauer E G 2001 Phys. Rev. B 63125317

[46] Carter G and Vishnyakov V 1996 Phys. Rev. B 5417647

[47] Ziberi B, Frost F, Höche T and Rauschenbach B 2005 Phys. Rev. B 72235310

[48] Davidovitch B, Aziz M J and Brenner M P 2007 Phys. Rev. B 76205420

[49] Shenoy V B, Chan W L and Chason E 2007 Phys. Rev. Lett. 98256101 